\title{
COMSEG TECHNIQUE FOR MRI KNEE CARTILAGE SEGMENTATION
}

\author{
SULAIMAN RIZA' ${ }^{1 @}$, DJASMIR MARLINAWATI ${ }^{2}$, MOHAMAD AMRAN MOHD FAHMI $^{3}$ \\ ${ }^{1}$ Institute of Visual Informatics, Universiti Kebangsaan Malaysia, 43600 Bangi, Selangor, Malaysia \\ ${ }^{2}$ Faculty of Information Science \& Technology, Universiti Kebangsaan Malaysia, 43600 Bangi, Selangor, Malaysia \\ ${ }^{3}$ Faculty of Science and Defense Technology, National Defence University of Malaysia, Kuala Lumpur, Malaysia \\ @E-mail: riza@ukm.edu.my (Corresponding author)
}

\begin{abstract}
Segmentation is one of important methods in medical images processing, particularly as it allows images to be analysed. The method used for segmentation depends on the image problem to be resolved. In this research, knee cartilage needs to be segmented to determine the level of the Osteoarthritis (OA) and for further treatment. Knee cartilage is a soft hyline sponge that is located at the end of the femur, tibia and patella bone to release friction during movement. OA is a knee cartilage problem wherein there is a thinning of the cartilage that results in a shift especially happening between femur and tibia bone causing discomfort and pain. Thinning of the knee cartilage is due to many factors such as age, body weight, genetic, accident, sport injury and extreme use such as physical work. OA can occur to a male or female, child or adult. The effects experienced by patients with OA are such as difficulty to walk, limited movement, and pain in the thin cartilage areas. Monitoring of patients' condition needs to be done to help reduce the problem and thereby enable specialists to perform the appropriate treatment. Imaging is a method used today to monitor the condition of patients with OA. Previous studies showed that MRI is a suitable method for monitoring the condition of patients with OA because of its advantages in visualising knee cartilage more clearly than other imaging methods. Thus, for segmenting the knee cartilage which as mentioned before is an important process in medical images processing, the MR images were selected based on many factors. Segmentation in this study was aimed to obtain the cartilage region to diagnose patient OA level. Various segmentation techniques have been developed by researchers in segmenting the knee cartilage region but they have been unable to segment precisely due to the thin structure of the knee cartilage, especially for patients with intermediate and severe OA. COMSeg technique was developed to segment knee cartilage, especially for those experiencing a normal and intermediate OA and try to implement it to severe OA. The development of this new technique takes into account the imaging method used, the images feature obtained so it can be suitable to process knee image and then selection of an appropriate technique to be applied to the selected images as input.
\end{abstract}

Keywords: MRI, knee cartilage, segmentation, osteoarthritis (OA)

\section{Introduction}

Osteoarthritis (OA) is a disease associated with the knee. OA is a condition where there is thinning, or loss of cartilage lines between the tibia and femur. The shift between two bones will cause the patient to experience pain, discomfort in the area of friction, limited or difficult movement. This situation not only applies to athletes or active individuals, normally it can happen to anyone, regardless of gender, age or background. To monitor and diagnose the state of OA patients, the structure of the knee image needs to be viewed and processed for further action. There are various imaging methods used in the world today including X-Ray,
CT Scan, ultra-sound and MRI to help doctors to analyse and diagnose various diseases $[1,2]$. Results of research conducted on a frequent and convenient method used for visualising the knee cartilage have shown that MRI is the most appropriate method in visualising the cartilage area [3]. Among the four imaging methods stated that are commonly used in medical, MRI is more suitable in visualising the knee structure. MRI has an abundance of advantages in OA imaging, high resolution in order to differentiate soft tissue, bone and even liquids with greater clarity [4, 5]. Imaging via MRI has minimal side effects compared to other imaging methods, therefore it is suitable to detect and monitor the progress of patients from time to time $[6,5]$.

Open Acces statement. This is an open-access article distributed under the terms of the Creative Commons AttributionNonCommercial 4.0 International License (https://creativecommons.org/licenses/by-nc/4.0/), which permits unrestricted use, distribution, and reproduction in any medium for non-commercial purposes, provided the original author and source are credited, a link to the CC License is provided, and changes - if any - are indicated. 
The issue of patient safety is of priority and important, which needs to be considered.

Segmentation is an important method in medical images processing, particularly as it allows images to be analysed [1, 7]. Determination of image segmentation techniques depends on the problem that needs to be resolved [8-10]. This is due to every part of the human body that has a density of muscle and different fluids. Various segmentation techniques have been developed by researchers to get the volume of knee cartilage. The knee cartilage segmentation method is divided into two types: edges-based and region-based. For this study, the region-based method is adopted as it is more suitable for gaining volume. Measurement of knee cartilage used to determine a person's level of OA is often done through the thickness, volume, curvature and surface area.

Segmentation is the process of distribution to particular parts of the image that are both similar in terms of intensity, texture and colour [1, 2] depending on the problem to be solved. In this research, segmentation of the region aimed at getting knee cartilage were analysed. The volume of segmentation results obtained will be used to determine a person's level of OA and further follow-up treatment can be carried out to help patients with OA. However, the segmentation of the knee cartilage is a difficult task due to the very thin cartilage structure, especially cartilage in patients with severe OA. Various image processing techniques have been developed by researchers to perform segmentation of cartilage and the results are still unsatisfactory [11]. Most of the results were only on performing segmentation of the normal knee cartilage.

\section{Problem statement}

Segmentation in knee cartilage images were very challenging due to the thin structure of the knee cartilage and also the intensity of the tissue surrounding the knee cartilage that makes it very difficult to identify and segment. In helping to simplify this segmentation process, there is some factor need to identify and concern before the segmentation can be implemented. Most of the problems faced by the researchers were during segmentation used as a guideline to find the factor need to be considered during pre-segmentation. First, the failure in choosing the right imaging method is the main cause for image processing to be difficult. Second is inaccurate image features selection that makes the image less clear or contain noise. And the last factor identified as a contributor to failure in segmenting properly the knee images is inaccuracy in choosing the method and technique processing to segment the image due to failure in choosing the right imaging method and image features.

\section{Methodology}

Based on the problem encountered and identified, we will discuss the solution which is not only focused on segmentation, but it starts with the right imaging method with the right features selection to visualise the knee image so that the segmentation process is easier.

\subsection{Selection of imaging method}

Therefore, the study needs to be done to enable the segmentation to be carried out at all OA stage in order to help and provide expertise in analysis and further treatment. Before performing image segmentation of the knee cartilage, an appropriate imaging method should also be taken into account. Inappropriate imaging method will cause a segmentation process to be difficult and affect the results of the segmentation. Based on previous studies, many researchers chose MRI as an imaging method for segmenting the knee cartilage due to its advantages compared to other imaging methods such as X-ray and CT scan. Changes to the knee cartilage and other soft tissues surrounding the knee cannot be seen by normal radiographs [3]. According to [2, 5, 12], MRI is widely used to visualise the image of the knee because with this method, the patient is at less risk exposing to harmful radiation and it can clearly visualise the soft tissue due to high resolution image. This is because the issue of patient safety is the main thing that should be emphasised especially involving repetitive analysis. For patients with OA, monitoring should be carried out from time to time depending on the patient's condition. Therefore, MRI is suitable to analyse and monitor the condition of patients with OA. Figure 1 shows the most suitable knee cartilage image which uses 3D DESS MRI with fat suppression in sagittal view with T2 weighted to be used to test the improvement technique developed.

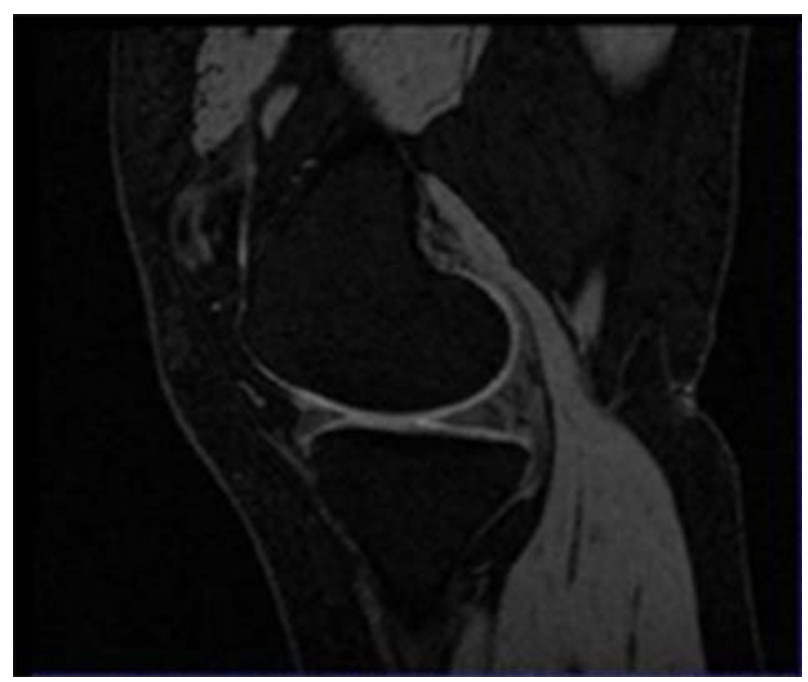

Fig. 1. MRI sagittal knee image with T2-weighted 


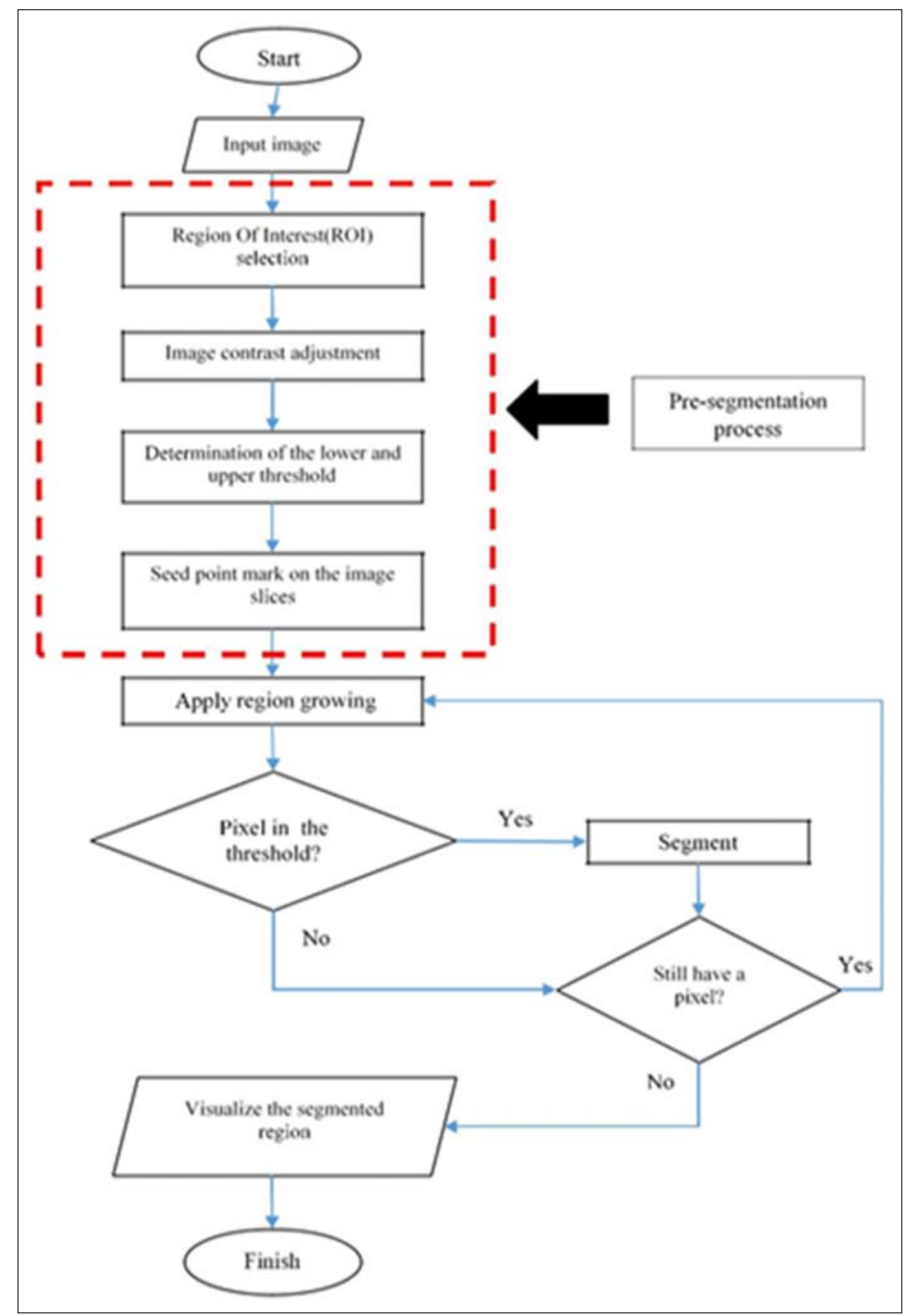

Fig. 2. Algorithm of COMSeg technique

\subsection{COMSeg technique}

According to Fig. 2, the process consists of two parts, namely, pre-segmentation and segmentation. Before the pre-segmentation is performed, the image of the knee will be read first. Then pre-segmentation process will be implemented. Pre-segmentation is an important process to ensure that segmentation process can be performed well [13]. The process of pre-segmentation of the image depends on the characteristics of the images obtained as mentioned before. If the images do not contain noise or contain less noise and the intensity of the pixels is clear, then the pre-segmentation becomes easier, and on the other hand, if the images contain noise and intensity of image pixels is less clear, then presegmentation is more difficult. The purpose of this research is to improve an image processing technique to perform segmentation of MR knee cartilage images, that consists of normal, intermediate and severe OA. The improvement technique developed is known as COMSeg technique. COMSeg stands for Contrast adjustment (CO), pixel Measurement $(\mathrm{M})$ using the lower and upper threshold and segmentation (Seg). Figure 2 shows the process involved 
in this technique. Even these algorithms used in these techniques were an improvement from existing method, but it is generated to suit the features of the image itself.

\subsection{Region of Interest selection (ROI)}

ROI selection is intended to shrink the region to the parts to be processed only. This allows the processing time of the image to be shortened and saves time because it fo-cuses on a specific region that needs to be processed. Figure 3 shows ROI selected in the red square area.

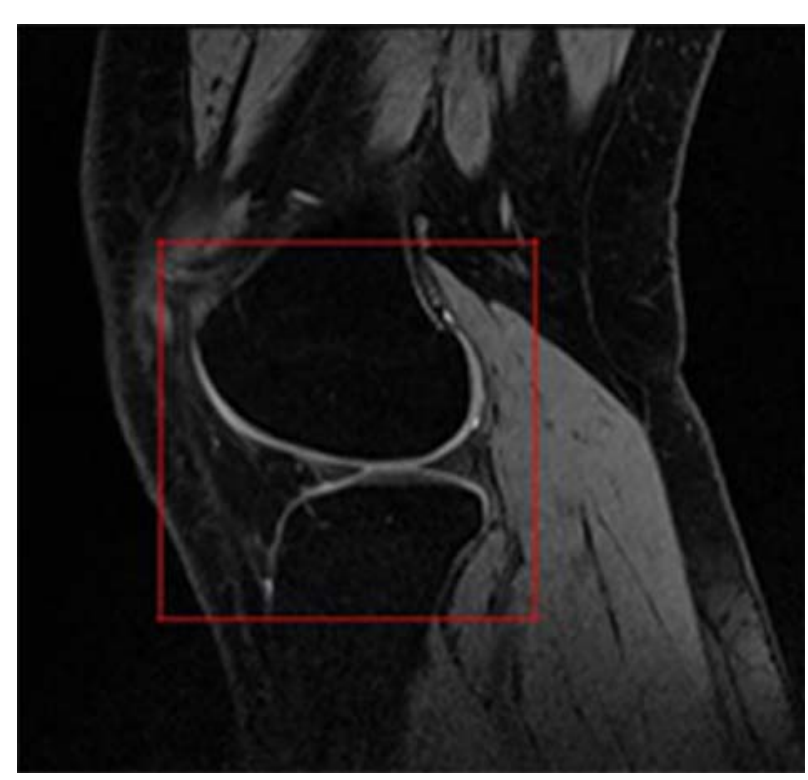

Fig. 3. Red square shows the ROI selected
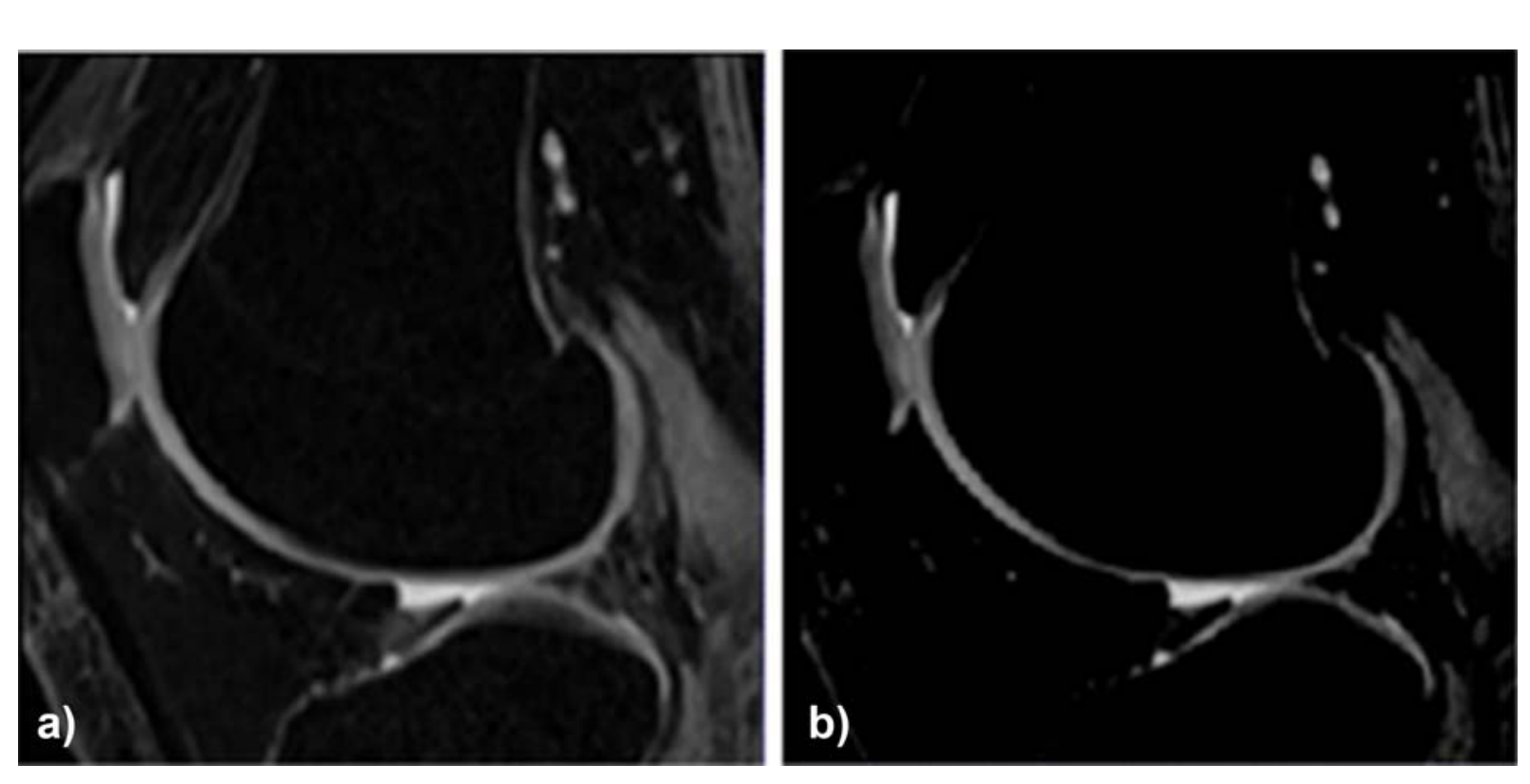

Fig. 4. a) Original image before contrast; b) Image after contrast adjustment

\subsection{Image contrast adjustment}

The next step is the contrast adjustment for regions that have been selected. Contrast adjustment is intended to increase the range of pixel intensity for the knee cartilage structure of the region. This is to distinguish the knee cartilage from other regions that are not needed, such as bone, muscle, ligaments and fluids. According to our investigation, the knee cartilage has a higher pixel intensity (brighter) than other surrounding tissues. Then, through the contrast adjustment with the specific value, knee cartilage can be seen more clearly while pixel intensity for structures other than knee cartilage is reduced. So from the adjustment of contrast, the light area will grow lighter and dark area will be getting darker. These processes really help to improve the quality of the image to be better compared to the image without contrast adjustment. However, there are still other structures near the knee cartilage, which have a pixel intensity brighter than the knee cartilage, which is not discussed by the majority of the researchers. The structure is known as the synovial fluid. Synovial fluid is located around the knee of the knee cartilage to protect it. The presence of this synovial fluid should be taken into account as it affects the segmentation result. By adjusting the contrast, other objects can be separated from the cartilage, but it cannot be separated from these synovial fluids because through contrast, as mentioned before, it makes the light area become lighter. So the intensity of the synovial fluid becomes lighter than the cartilage. Therefore, another process is required to separate the knee cartilage and other objects, especially this synovial fluid. The next process is how to separate the cartilage from this synovial fluid and balance the other remaining objects. The 
Table 1. Lower and upper threshold value for cartilage

\begin{tabular}{lccc}
\hline & Other object & Cartilage & Synovial fluid \\
\hline Lower threshold & $<165$ & 165 & \\
Upper threshold & & 300 & $>300$ \\
\hline
\end{tabular}

difference between the original image and the adjusted image contrast is shown in Fig. 4.

\subsection{Determination of Lower and Upper Threshold}

The separation between synovial fluid, knee cartilage and other remaining objects is done by setting the lower and upper threshold. These values are fixed and suitable only for cartilage intensity and done under expert monitoring to make sure only the cartilage is segmented. The lower and upper value implemented during segmentation is using region growing method. When applying the region growing, knee cartilage will be segmented within the upper and lower threshold range value. Table 1 indicate the lower and upper threshold value use for the images selected in this research. This value is tested to all OA levels under expert monitoring.

Region growing technique will read the value range and compare to image pixel. This process is repeated until no more pixels in that range value need to be processed.

\subsection{Seed point mark on the image slices}

After setting the lower and upper threshold value, the next process is to place the seed point into knee cartilage area as a sign to start growing region. Seed point mark is compulsory for region growing method. This seed point should be located in several places on the image slices to increase processing speed as it occurs from various directions and thus can save time to process the image. In addition, it is also to avoid regional cuts and are not processed especially for the severe OAs. The disconnected region is intended to occur primarily on the patient's knee cartilage with severe $\mathrm{OA}$. Knee cartilage with severe OA are increasingly difficult to segment not only due to the structure of the region being thin but there are also missing cartilage areas. So, to prevent this occuring, it is important to put on a few seed points. Seed point marking is not necessarily similar between one patient and another because of the structure of the knee, size and condition of knee cartilage that differ from one individual to another.

\subsection{Cartilage segmentation}

After placing the seed point, the next process was a very important process in image processing, called segmentation before the image can be analysed. The segmentation process will use a region growing method to get the region of the knee cartilage. This segmentation process will occur simultaneously for all the seed points specified earlier. During the execution of region growing, as mentioned before we will use the lower and upper threshold value to be applied to the seed point. This process will be repeated until there
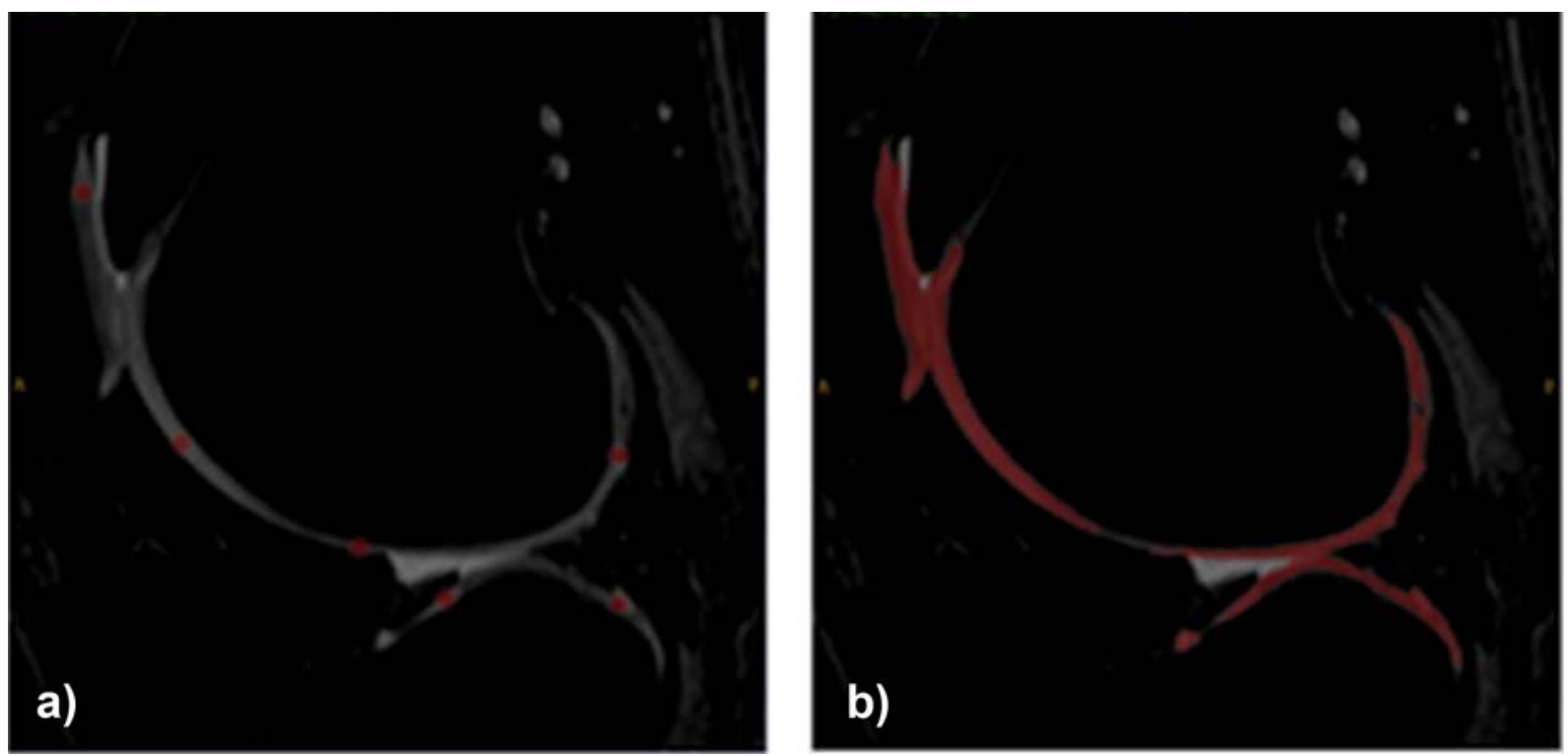

Fig. 5. a) seed point mark, b) segmentation result for the normal OA 
are no more pixels within the range of the lower and upper threshold to be processed.

\section{Result}

Figures 5, 6 and 7 show that the process starts with a) seed point mark and results of segmentation. Figure 5 for normal OA, Fig. 6 for intermediate OA and Fig. 7 for severe OA. Image a) shows the seed point mark and image b) shows the result of the segmentation. The results obtained from image after segmentation indicate that these COMSeg techniques can be used and applied to the MR image of normal and intermediate OA precisely. While for the severe OA, the re- sult visualises that this COMSeg technique can also be used for severe OA but sometimes still problem occur.

At the same time, Figs 8, 9 and 10, respectively, show the visualisation of the segmentation result in 3D. Figure 8 presents the result for normal OA, Fig. 9 for intermediate OA and Fig. 10 for severe OA. From the three visualisations we can see a significant change between normal, intermediate and severe OA.

These techniques apply to 90 data of OA patients which consist of 30 normal OA, 30 intermediate OA and 30 severe OA. Out of the 3 categories of OA level, only data from severe OA have some problem during segmentation for certain image. Table 2 shows the average volume for males and Table 3 shows
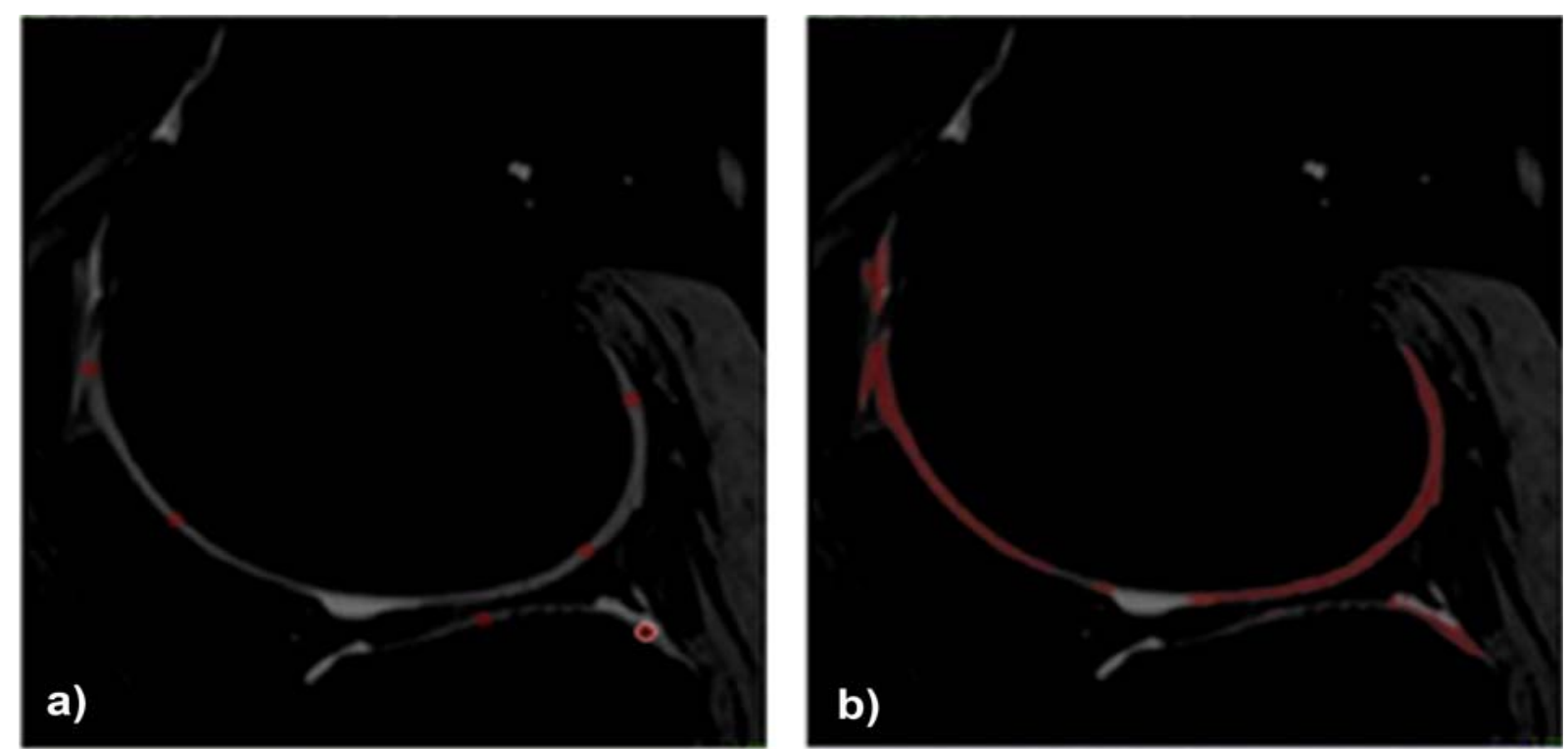

Fig. 6. a) seed point mark, b) segmentation result for the intermediate OA
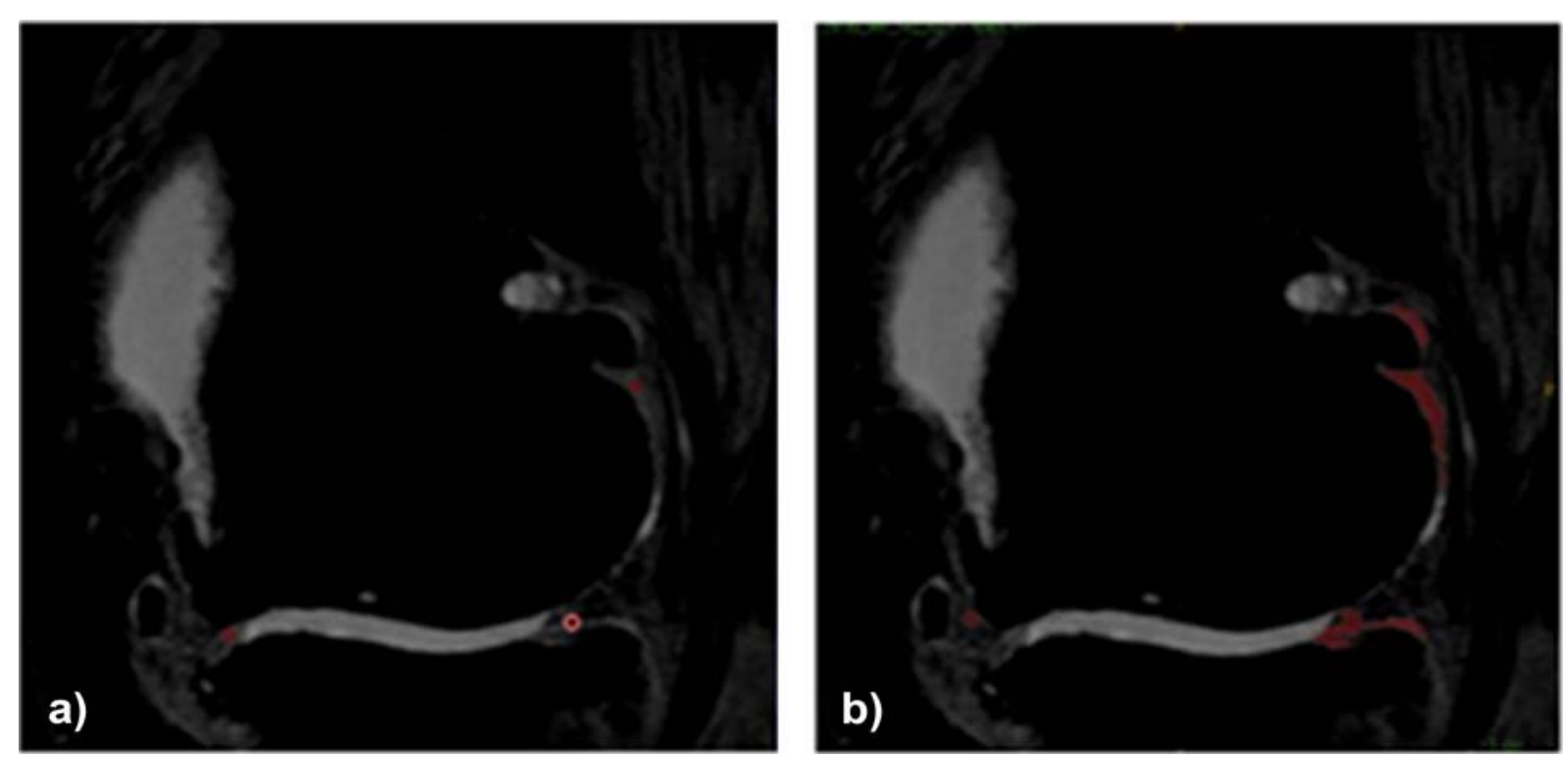

Fig. 7. a) seed point mark, b) segmentation result for the severe OA 


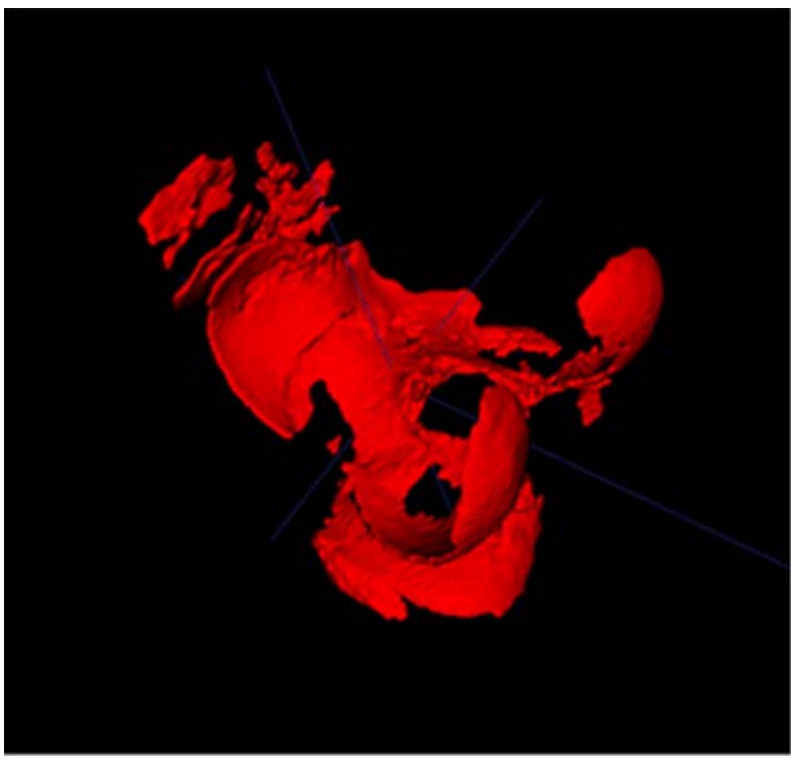

Fig. 8. Visualisation of normal OA knee cartilage

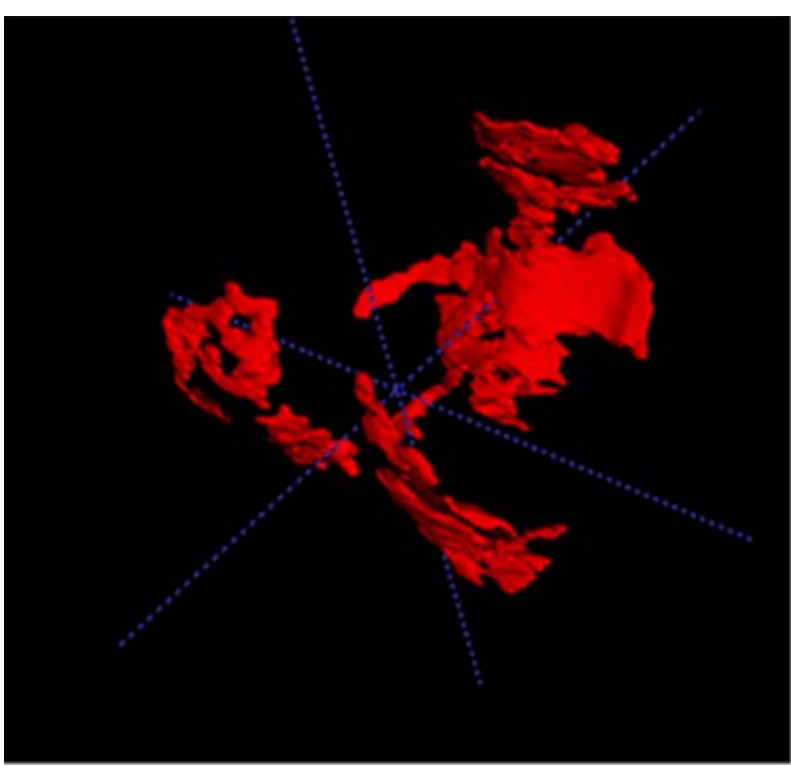

Fig. 10. Visualisation of severe OA knee cartilage

the average volume for females for all three categories of OA level. This value can be used to determine the category of OA. For males, the average cartilage volume for normal OA ranges from $\pm 11,000 \mathrm{~mm}^{3}$ to $\pm 15,000 \mathrm{~mm}^{3}$, intermediate knee ranges from $\pm 8,000 \mathrm{~mm}^{3}$ to $\pm 11,000 \mathrm{~mm}^{3} \mathrm{~cm}$ and severe OA ranges from $\pm 5,000 \mathrm{~mm}^{3}$ to $\pm 8,000 \mathrm{~mm}^{3} \mathrm{~cm}$, whereas for females, mean cartilage volume for normal OA ranges from $\pm 8,000 \mathrm{~mm}^{3}$ to $\pm 11,000 \mathrm{~mm}^{3}$, intermediate knee ranges from $\pm 6,000 \mathrm{~mm}^{3}$ to $\pm 8,000 \mathrm{~mm}^{3} \mathrm{~cm}$ and severe OA ranges from $\pm 3,000 \mathrm{~mm}^{3}$ to $\pm 6,000 \mathrm{~mm}^{3} \mathrm{~cm}$.

This comparison shows in a graph in Fig. 8 to visualise the comparison easily. From the graph, this proved that patients with intermediate OA and severe

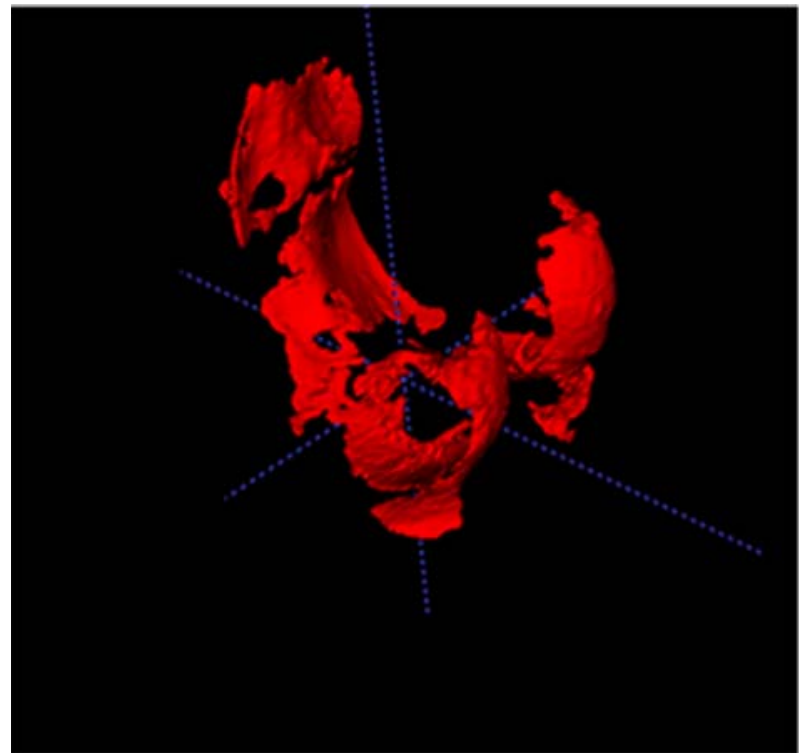

Fig. 9. Visualisation of intermediate OA knee cartilage

Table 2. Volume range for male cartilage

\begin{tabular}{ccl}
\hline $\begin{array}{c}\text { Minimum value } \\
\left(\mathrm{mm}^{3}\right)\end{array}$ & $\begin{array}{c}\text { Maximum value } \\
\left(\mathrm{mm}^{3}\right)\end{array}$ & OA value \\
\hline $\pm 11,000$ & $\pm 15,000$ & Normal \\
$\pm 8,000$ & $\pm 11,000$ & Intermediate \\
$\pm 5,000$ & $\pm 8,000$ & Severe \\
\hline
\end{tabular}

Table 3. Volume range female cartilage

\begin{tabular}{ccl}
\hline $\begin{array}{c}\text { Minimum value } \\
\left(\mathrm{mm}^{3}\right)\end{array}$ & $\begin{array}{c}\text { Maximum value } \\
\left(\mathrm{mm}^{3}\right)\end{array}$ & OA value \\
\hline $\pm 8,000$ & $\pm 11,000$ & Normal \\
$\pm 6,000$ & $\pm 8,000$ & Intermediate \\
$\pm 3,000$ & $\pm 6,000$ & Severe \\
\hline
\end{tabular}

OA had less knee cartilage thickness in comparison to normal OA knee. There is significantly different volume between these three categories of OA. The difference is quite significant for male cartilage volume for all categories compared to women. This is because of the thinner structure of the female cartilage.

\section{Discussion}

COMSeg technique is a rather simple technique developed to help experts to segment the knee cartilage. Results of the segmentation from the knee cartilage are intended to get the volume that can be used to analyse the OA levels for follow up treatments. However, there is little difficulty in segmenting severe OA level due to the cartilage structure being difficult to identify. However, this technique has been applied to an image 


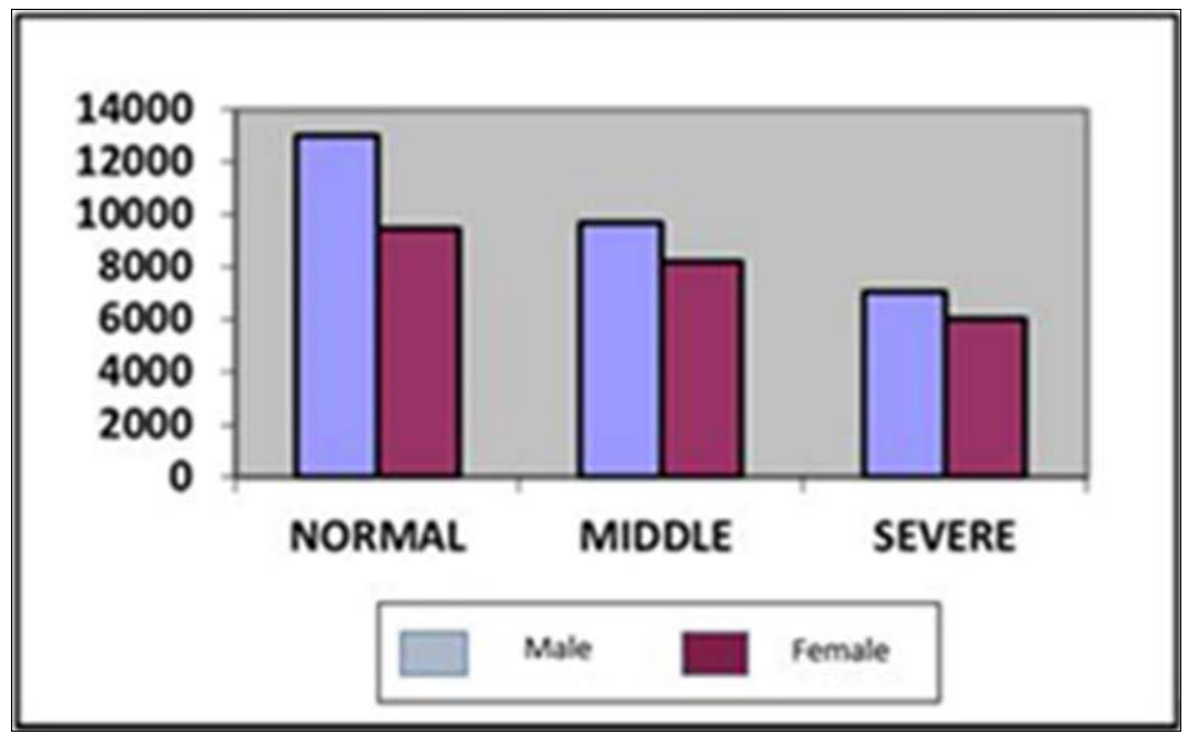

Fig. 11. Volume difference for all three categories of OA for males and females

of severe OA to test the ability of the resulting technique, segmentation results showed that some severe OA images cannot be segmented precisely compares to normal and intermediate OA.

This is because there are many areas in severe OA images that are cut off and too thin. Therefore, the developed COMSeg techniques is suitable to be applied in both normal and intermediate OAs. Improvements need to be made so that it can be applied to patients suffering from severe OA. To date, there are a limited number of studies published about calculation of the volume of cartilage. Most studies are doing segmentation without separating synovial fluid that exists near the cartilage. Even if the volume of the synovial fluid is too small, it still affects the cartilage volume results. There is no research proving that synovial fluid relates to OA disease. Some of the studies apply the technique developed to normal OA levels only and have a problem if applied to a problem cartilage image data, while there is a segmentation technique developed to get the surface area and cannot be used to determine the level of OA. Also, previous published studies reporting on measuring cartilage thickness which were less precise in determining the patient OA level because this method focused on certain angel. In addition, by measuring the thickness, there is less information that can be used for further treatment.

\section{Conclusion}

The measurement of the knee cartilage volume using 3D DESS MRI with fat suppres-sion is a potential, reproducible and reliable tool for determination of knee cartilage vol-ume in order to monitor disease progression and monitoring treatment response in $\mathrm{OA}$ patients. Although the method used in COMSeg tech- nique are already available, still, in this research, the techniques have been adapted to suit the needs of consumers (medical expert). We not only focus on segmenting technique, which is the main part of medical image processing, but also emphasise other issues such as an appropriate imaging method and features in visualising the cartilage image to be processed and pre-segmentation method. Mostly the researcher only uses the data given to test the technique developed without emphasising the issue stated.

\section{References}

[1] Choudhari S., Biday S. (2013), Medical Image Segmentation, International Journal of Advanced and Innovative Research, 2(10), 399-403.

[2] Dougherty G. (2009), Digital Image Processing for Medical Applications. 1st ed., Cambridge University Press, New York.

[3] Mohd Khairul A. (2015), MR Imaging of Knee Articular Cartilage Thickness: Correlation with Severity of Osteoarthritis.

[4] Sanjeevakumar K., Ravikumar K. M., Harini D. G. (2013), Measurement of Cartilage Thickness for Early Detection of Knee Osteoarthritis (KOA). In: Point-of-Care Healthcare Technologies (PHT), pp. 208-211. IEEE, Bangalore, India.

[5] Mallikarjunaswamy M. S., Mallikarjun S. H. (2012), Knee joint articular cartilage segmentation, visualization and quantification using image processing techniques: A review. International Journal of Computer Applications, 42, 36-43.

[6] Tamez-Peña J. G., et al. (2012), Unsupervised segmentation and quantification of anatomical knee features: Data from the Osteoarthritis Initiative. IEEE Trans. Biomed. Eng., 59, 1177-1186. 
[7] Bhabhor C. H., Upadhyay A. B. (2013), Performance analysis for image segmentation of various edge detection techniques. Journal of Information, Knowledge and Research in Electronics and Communication Engineering, 2(2), 399-404.

[8] Chaudhary A., Gulati T. (2013), Segmenting digital images using edge detection. International Journal of Application or Innovation in Engineering \& Management, 2(5), 319-323.

[9] Pham D. L., Xu C., Prince J. L. (2000), A survey of current methods in medical image segmentation. Annual Reviews of Biomedical Engineering, 2, 315-337.

[10] Gonzalez, R. C., Woods R. E. (2008), Digital Image Processing. 3rd edn. Pearson Prentice Hall, New Jersey.
[11] Fripp J, Crozier S, Warfield SK, Ourselin S. (2010), Automatic segmentation and quantitative analysis of the articular cartilages from magnetic resonance images of the knee. IEEE Trans. Med. Imaging, 29(1), 55-64.

[12] Teichtahl A. J., Wluka A. E., Davies-Tuck M. L., Cicuttini F. M. (2008), Imaging of knee osteoarthritis. Best Pract. Res. Clin. Rheumatol., 22(6), 1061-1074

[13] Ngo Q. L. (2011), Image processing on medical application: Automatic methods to calculate the area of an articular cartilage on a magnetic resonance image. Research Master Thesis, University of Tasmania, Australia. 